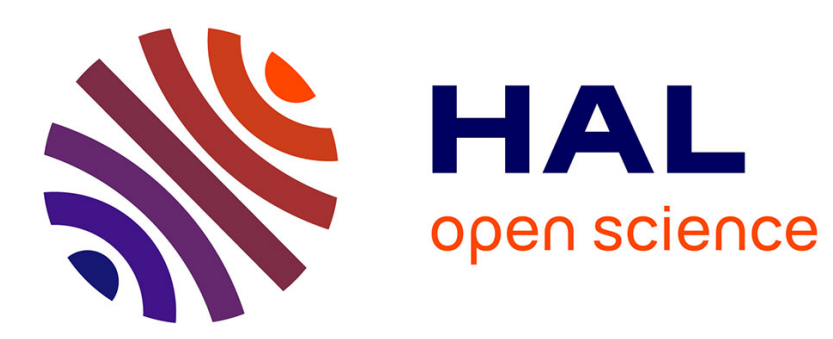

\title{
L'histoire du bilinguisme en Ukraine et son rôle dans la crise politique d'aujourd'hui
}

Natalya Shevchenko

\section{To cite this version:}

Natalya Shevchenko. L'histoire du bilinguisme en Ukraine et son rôle dans la crise politique d'aujourd'hui. Cahiers Sens Public, 2014, n 17-18 (1), pp.203. hal-02442738

\section{HAL Id: hal-02442738 \\ https://hal.univ-lyon2.fr/hal-02442738}

Submitted on 21 Feb 2020

HAL is a multi-disciplinary open access archive for the deposit and dissemination of scientific research documents, whether they are published or not. The documents may come from teaching and research institutions in France or abroad, or from public or private research centers.
L'archive ouverte pluridisciplinaire HAL, est destinée au dépôt et à la diffusion de documents scientifiques de niveau recherche, publiés ou non, émanant des établissements d'enseignement et de recherche français ou étrangers, des laboratoires publics ou privés. 


\section{L'histoire du bilinguisme en Ukraine et son rôle \\ dans la crise politique d'aujourd'hui}

\section{Natalya SHEVCHENKO}

Centre de Recherche en Terminologie et Traduction (CRTT), Université Lumière Lyon 2

\section{Résumés}

La possession d'une langue commune et unique est considérée comme la condition principale de formation et de consolidation d'une nation. En Ukraine, jusqu'à récemment, la conscience nationale s'appuyait essentiellement sur la conscience linguistique et culturelle de la nation. Les événements des années 2013 et 2014 ont renforcé et mis en avant la conscience politique des Ukrainiens qui sont désormais obligés de se battre pour l'indépendance et l'intégrité territoriale de leur pays.

L'article retrace brièvement I'histoire de la formation et de l'évolution de l'ukrainien et de son interaction avec le russe dès son origine jusqu'à aujourd'hui. Il décrit le rôle de la langue dans le conflit actuel entre la Russie et l'Ukraine.

Possession of a single, common language is considered as the main requirement for the formation and consolidation of a nation. In Ukraine, until recently, the national consciousness was based mainly on the nation's linguistic and cultural charactertistics. The events of 2013 and 2014 have strengthened and highlighted the political consciousness of Ukrainians, who are now forced to fight for the independence and territorial integrity of their country.

This article outlines a brief history of the Ukrainian language's formation and evolution alongside its interaction with Russian from its very beginnings to the present day, as well as exploring the role of language in the present conflict between Russia and Ukraine.

Наличие общего и единого языка является обязательным условием для формирования и консолидации любой нации. В Украине до недавнего времени национальное сознание опиралось главным образом на лингвистические и культурные особенности нации. События 2013-2014 гг. вывели на первый план политическое сознание украинцев, которые, несмотря на языковую разрозненность внутри страны, борются за независимость и территориальную целостность своего государства.

В статье изложена краткая история становления украинского языка и его взаимодействия с русским языком от истоков до настоящего времени, а также роль лингвистического фактора в настоящем конфликте между Россией и Украиной.

\section{Mots-clés}

Bilinguisme ukrainien-russe, identité nationale, histoire de l'ukrainien, conflit politique, révolution 2013-2014 en Ukraine

\section{Introduction}

La conscience nationale s'appuie essentiellement sur la conscience linguistique et culturelle de la nation. Sans identité linguistique, même la culture d'origine peut se dissoudre dans la culture de la langue dominante, au risque pour la nation, en tant que telle, de se retrouver en danger de disparition. La langue reste donc l'une des marques identitaires de chaque nation. La langue nationale joue un rôle primordial dans la formation d'une nation et remplit, après sa fonction primaire qui est la fonction de communication, deux fonctions principales, unificatrice et séparatrice : rassembler la population au sein d'un pays et la distinguer de l'extérieur, de ses voisins. 
La possession d'une langue commune est considérée comme la condition principale de l'existence d'une nation. Une nation peut coïncider avec l'État, peut exister sans État et peut faire partie, à l'égal d'autres nations, d'un État fédéral.

Il y a 10 ans, dans son ouvrage sur le rapport entre la langue et la politique en Ukraine, la sociolinguiste renommée Larysa Masenko avait mis la société ukrainienne en garde en anticipant le conflit actuel : «L'extension de deux ou plusieurs langues dans un pays témoigne [...] d'un équilibre incertain qui risque un jour soit d'amener au monolinguisme, soit à la division du pays par le critère linguistique »(2004a:19). Cet avis, au premier abord catégorique, s'avère difficile à réfuter si l'on analyse la situation dans d'autres pays qui sont bi- ou même multilingues. Les exemples de la Belgique et du Canada d'un côté, de la Serbie et de la Croatie de l'autre, pour n'en citer que quelquesuns, confirment le principe du monolinguisme nécessaire pour une nation forte et unie. Néanmoins, les pays bilingues existent, certes, fondés sur des principes différents, mais leur existence plus ou moins réussie prouve tout de même la possibilité d'une telle expérience.

Quel est alors le ciment des pays bilingues ? Le bilinguisme canadien ainsi que le cas belge, selon McRoberts (Lacorne \& Judt, 2002:150-151, 190), politologue canadien qui a analysé la situation québécoise, le fédéralisme canadien et étudié les questions d'ordre constitutionnel, reposent, entre autres, sur le principe de la ségrégation de l'espace des territoires linguistiques. Cette territorialisation permet la cohabitation plus ou moins paisible de deux, voire plusieurs cultures sur le territoire d'un pays, mais ne résout pas le problème du bilinguisme national. Au contraire, elle amène souvent à l'unilinguisme régional.

Le seul exemple d'un pays européen avec une société polyphonique réussie regroupant plusieurs nations semble être la Suisse, où la force nationale est fondée sur l'unité dans la diversité. Windisch, qui traite du cas suisse (op.cit:227-259), distingue trois composantes dont la présence simultanée et conjointe est nécessaire pour une cohabitation entre communautés culturelles et linguistiques différentes au sein d'un même État: identité culturelle, communication interculturelle, et culture politique commune à toutes les communautés linguistiques et culturelles (ibid::228). La Suisse possède quatre langues nationales (allemand, français, italien et romanche) et donc quatre communautés culturelles différentes. La cohésion du pays, explique Windisch (ibid.:229), ne vient pas du plurilinguisme de ses habitants, mais plutôt du fait que tous les Suisses partagent une culture politique commune, notamment la démocratie directe et le fédéralisme. Évidemment, la Suisse connaît un certain nombre de difficultés liées à son pluriculturalisme, mais elles sont minimes par rapport aux contrariétés submergeant d'autres pays multilingues. Le cas suisse prouve mieux que les autres la possibilité d'une cohabitation interculturelle prospère au sein d'un État, et nous mène à une conclusion évidente: les problèmes des pays bi- ou plurilingues ne sont jamais des problèmes purement linguistiques ou culturels, mais des problèmes principalement politiques. Le cas ukrainien analysé en détail dans les pages qui suivent en est une preuve. 


\section{Les origines}

La langue ukrainienne est une des langues slaves qui constituent une des branches les plus importantes et les plus homogènes de la famille indo-européenne. Elle appartient avec trois autres langues - le biélorusse, le russe et le ruthène - au groupe du slave oriental (ou de l'Est). Aujourd'hui I'ukrainien est parlé par 41 millions de locuteurs (Tableau 1). Ce chiffre est polémique car les données

Tableau 1 : La famille indo-européenne Source : Leclerc (2007)

\begin{tabular}{|c|l|}
\hline $\begin{array}{c}\text { Sous-groupes du groupe Slave } \\
\text { (plus de } 315 \text { millions }^{1} \text { de locuteurs } \\
\text { dans le monde) }\end{array}$ & \multicolumn{1}{|c|}{ Langues } \\
\hline Slave occidental & $\begin{array}{l}\text { polabe (langue morte) } \\
\text { polonais (44 millions) } \\
\text { tchèque }(12 \text { millions) } \\
\text { slovaque }(5,6 \text { millions }) \\
\text { sorabe }(70 \text { 000) }\end{array}$ \\
\hline Slave méridional & $\begin{array}{l}\text { slavon (langue morte) } \\
\text { croate } \\
\text { monténégrin }=\text { serbe } \\
\text { bosniaque } \\
\text { slovène (2,2 millions) } \\
\text { bulgare }(9 \text { millions) } \\
\text { macédonien }(2 \text { millions })\end{array}$ \\
\hline Slave oriental & $\begin{array}{l}\text { russe (170 millions) } \\
\text { biélorusse }(10,2 \text { millions }) \\
\text { ukrainien (41 millions) } \\
\text { ruthène (125 000) }\end{array}$ \\
\hline
\end{tabular}

qu'on trouve sont très variées en fonction de la source. Ainsi, Lewis dans son Encyclopédie des langues du monde (2009) donne 37 millions de locuteurs partout dans le monde. Selon Malherbe (1995:184), les locuteurs de l'ukrainien sont 52 millions. Cet écart de 15 millions est difficilement explicable vu que les dates de la récolte des données sont très rapprochées : 1993 pour Lewis et et 1995 pour Malherbe. Le chiffre de 41 millions cité par Leclerc (2007) me paraît le plus proche de la réalité compte tenu du nombre de la population générale en Ukraine (47,2 millions en 2005), le pourcentage des Ukrainiens dans le pays $(77,8 \%=37,5$ millions, selon I'Office ukrainien des statistiques) et de fortes diasporas ukrainiennes aux États-Unis (2 millions) et au Canada (1 million), plus d'autres diasporas partout dans le monde.

L'alphabet ukrainien, tout comme les alphabets russe, biélorusse, serbe, bulgare et macédonien est cyrillique. Jadis, l'ukrainien, le russe et le biélorusse constituent une seule langue, l'ancien slave ou slavon. Il s'agit à l'origine du bulgare ancien, importé en Rous de Kyiv avec le christianisme, qui remplit une fonction de langue écrite analogue à celle du latin en Europe occidentale, et s'oppose

\footnotetext{
${ }^{1}$ Pourtant, si I'on additionne les chiffres donnés par Leclerc, on obtient 316,8 millions de locuteurs.
} 
toujours à la langue parlée, l'ancien ukrainien/russe (qui s'écrit aussi). Ainsi, déjà à cette époque, on peut parler du bilinguisme ou de la diglossie entre le langage parlé (I'ancien ukrainien) et la langue écrite (le slavon). Ce slavon écrit sert initialement à traduire des textes religieux du grec. Il demeure présent en Ukraine comme langue ecclésiastique d'abord, et ensuite comme standard littéraire, figé jusqu'à la fin du XVII ${ }^{\mathrm{e}}$ siècle. Les trois langues apparentées commencent à se fragmenter vers le XII siècle. Les premières grammaires d'ukrainien ancien apparaissent vers le début du XVI ${ }^{\mathrm{e}}$ siècle, tandis que le premier dictionnaire en langue ukrainienne voit le jour un peu plus tard, en 1596 : il s'agit du Leksis de Lavrentij Zyzanija, dictionnaire qui accompagne sa grammaire et donne des équivalents ukrainiens aux mots du slave ecclésiastique. Cependant, la langue ukrainienne moderne ne sera en place que deux siècles plus tard.

\section{Entre deux empires : la constitution de la langue littéraire}

À partir de 1654, quand l'Ukraine est divisée entre la Russie et la Pologne, I'ukrainien de la partie Centre-Est de I'Ukraine se limite à la langue populaire parlée par les paysans. Par la suite, les réformes de Pierre le Grand interdisent l'emploi de l'ukrainien à l'église et dans les textes religieux. Catherine II achève la suppression de I'ukrainien littéraire de l'usage des Ukrainiens de I'Est. La russification massive des villages ukrainiens, l'usage du russe comme seule langue de communication dans les organismes officiels, le passage au russe de l'intelligentsia ukrainienne et de la majorité des dirigeants cosaques produisent leur effet très rapidement : déjà à la fin du XVIII siècle, l'ukrainien disparaît de l'usage officiel sur le territoire russe de l'Ukraine.

Le XVIII ${ }^{e}$ siècle devient un siècle de transformation et d'enrichissement du russe : sa forme écrite avec le fondement religieux et sa forme orale avec la variante populaire se réunissent dans une seule et unique langue russe. Lomonossov fonde l'université de Moscou et propose, entre autres, dans le but de normaliser la langue russe, une distinction de trois styles: élevé, moyen et bas. Les principes stylistiques de Lomonossov seront appliqués par Karamzine, qui sera le premier à écrire en russe littéraire moderne et non en slavon. Pouchkine, Tourgueniev, Gogol, Dostoïevski le suivront et apporteront chacun leur pierre à l'édifice de la langue russe. Ainsi, le XIXe voit le russe standard se fixer et parvenir à l'apogée de son épanouissement.

A cette époque, la langue ukrainienne ne dispose, elle, que de sa forme populaire. Ce sera cette langue populaire, orale avant tout, qui sera à la base de la nouvelle langue ukrainienne standardisée. Ainsi, Ivan Kotljarevs'kyj (1769-1838) utilise cette substance populaire et folklorique pour créer son style dans la littérature, fondant la nouvelle langue littéraire. Il faut noter qu'il y a une grande différence dans ces termes en français et en ukrainien. Langue littéraire en ukrainien ne signifie pas forcément la langue de la littérature mais la langue nationale, générale, normalisée, la langue standard, appelée aussi le « standard écrit ». Le poème Enejida (1798) de Kotljarevs'kyj englobe toute la richesse de l'humour populaire de l'époque, reflète les traditions et le quotidien des Ukrainiens. Le ton satirique de Kotljarevs'kyj est repris par d'autres écrivains, comme Hulak-Artemovs'kyj, Hrebinka, Kvitka-Osnov'janenko, etc. Ce dernier, fondateur de la prose ukrainienne, prouve par son œuvre la possibilité d'écrire en ukrainien, et pas seulement des textes comiques. 
Le plus grand poète ukrainien, Taras Shevchenko (1814-1861), engendre ensuite la langue littéraire ukrainienne telle qu'elle est aujourd'hui. Il enrichit le langage de l'époque, figé, littéraire et peu naturel, avec un lexique familier et populaire. L'œuvre de Shevchenko contribue au perfectionnement de la langue ukrainienne, à son épanouissement lexical et stylistique : synonymie, néologie, rythmique, etc. Sa poésie étoffe la langue, la fait connaître en dehors de l'Ukraine. Son nom ne représente pas seulement la littérature, il est aussi devenu un symbole de la culture ukrainienne.

T. Shevchenko sera suivi de toute une pléiade d'écrivains qui ont tous contribué à l'épanouissement de la langue ukrainienne : Kuliš, Lesja Ukrajinka, Franko, Kocjubyns'kyj.

La deuxième moitié du XIXe et le début du XXe siècle sont caractérisés par un développement de divers styles littéraires, ainsi que de la terminologie scientifique et du langage de la presse. Cependant, cette expansion linguistique s'opère d'une façon inégale suivant les différentes parties du pays, divisé à cette époque entre deux empires : russe et austro-hongrois. C'est dans la partie austrohongroise de l'Ukraine, en particulier à Lviv et à Tchernivtsi, que les styles officiel, scientifique et celui de la presse se sont formés. Parallèlement à l'émergence des styles, on voit apparaître dans les villes des koinès ${ }^{2}$ à base ukrainienne : des langages particuliers de l'intelligentsia, des entrepreneurs, des artisans, etc. Ces changements linguistiques ont résulté de l'urbanisation qui a activé l'apparition simultanée de différents milieux citadins parlant ukrainien.

Ainsi, si I'Ukraine austro-hongroise avançait progressivement dans l'établissement des normes littéraires - et même dans la création de la terminologie scientifique en ukrainien - I'Ukraine de l'Est subissait des décrets tsaristes interdisant l'un après l'autre la langue ukrainienne. L'un des décrets les plus violents de cette époque, est la circulaire secrète du 8 juillet 1863 du ministre de l'Intérieur Valuev, qui refusait toute reconnaissance de la langue ukrainienne en tant que telle (« il n'y a jamais eu de langue ukrainienne, il n'y en a pas maintenant et il n'y en aura jamais ») et la proscrivait sur le territoire de l'Empire. L'enseignement en ukrainien dans les écoles est également interdit (jusqu'en 1917). Même le nom ukrainien est prohibé et remplacé par un autre - malorusskij, « petit-russe ». Par la suite, toute littérature en ukrainien est interdite d'accès en Ukraine de l'Est. Cette période d'« allergie à tout ukrainien » dura de 1863 à 1905.

En 1905, sous l'influence des premiers mouvements révolutionnaires en Russie, les publications en ukrainien et les associations culturelles ukrainiennes sont à nouveau autorisées. La presse ukrainienne bénéficie aussi d'une courte période favorable. Entre les deux révolutions et ensuite, avec I'indépendance de I'Ukraine en 1917, de nombreux enrichissements surviennent dans le système de style, dans la traduction, ainsi que dans le domaine de la lexicographie : dictionnaire ukrainienallemand de Želexivs'kyj et Nedil's'kyj (1885-1886), dictionnaire d'ukrainien de Hrinčenko (19071909), grammaires de Kryms'kyj (1907-1908), de Simovyč (1918, 1919), etc. Ces ouvrages contribuent à la normalisation et la codification des normes de I'ukrainien littéraire. Une large polémique a lieu autour de l'alphabet et de l'orthographe. Il faut noter que cela a été un moment de déblocage entre l'Ouest et l'Est de l'Ukraine, ce qui a permis un échange de connaissances entre les

\footnotetext{
${ }^{2}$ Toute langue commune qui se superpose à un ensemble de dialectes ou de parlers sur une aire géorgraphique donnée (Dubois \& al., 2002:262).
} 
deux parties du pays, en particulier, les connaissances accumulées en Galicie et en Bucovine durant les trente années précédentes. Le lexique en provenance de ces régions a ainsi contribué à la formation des styles scientifique et journalistique qui feront, par la suite, partie de la langue générale standardisée.

Traditionnellement, on attribue l'origine du standard littéraire ukrainien à la région de Kyiv et Poltava. Shevelov (1993:947), lui, parle d'une base bi-dialectale de l'ukrainien standard d'aujourd'hui : les dialectes du sud-est, en particulier du sud de Kyiv et des régions de Tcherkassy et de Poltava d'un côté, et les dialectes du sud-ouest avec Lviv comme centre culturel de l'autre. L'influence des dialectes sud-ouest se manifeste surtout en lexique mais aussi en phonologie et ceci depuis le Moyen-Âge. Le pic de cette interaction correspond à la fin du XIX et au début du XXe siècle. Ainsi, la théorie commune sur l'origine de l'ukrainien littéraire est remise en question par ce chercheur, même s'il ne nie pas que la contribution orientale soit nettement plus importante.

\section{Les années 1920 : ukrainisation}

Le standard littéraire en ukrainien s'est définitivement formé dans les années 1920, à l'époque de I'ukrainisation. Une courte période d'indépendance (1917-1920) est interrompue par la création de la République socialiste soviétique d'Ukraine en 1922. L'Ukraine est ensuite annexée à I'URSS. Mais la politique de l'État soviétique par rapport aux minorités nationales se distinguait de celle de l'Empire. En effet, la position des communistes à l'époque étant encore très instable dans un État multiethnique, leur politique dans les questions nationales devait être souple. Lénine comprenait que pour l'affirmation du pouvoir soviétique en Ukraine, il fallait faire des concessions dans la question nationale. Les diverses nationalités de I'URSS obtiennent le droit d'utiliser leurs langues dans les écoles et les administrations locales. La nécessité de savoir parler l'ukrainien au travail s'impose alors. La langue ukrainienne est proclamée langue officielle de I'Ukraine, tandis que le russe obtient un statut de langue générale de communication en URSS. Concernant les minorités nationales en Ukraine, la politique de l'État soviétique sur l'égalité de toutes les langues nationales aboutit à la création de quartiers nationaux avec des écoles, des théâtres, des journaux dans les langues des minorités russe, bulgare, allemande, juive, grecque, polonaise, etc.

Rappelons qu'à cette époque, Staline partageait les opinions politiques de Lénine : « Il est [...] clair que la nation ukrainienne existe réellement et que le développement de sa culture est un devoir des communistes. On ne peut pas aller à l'encontre de I'histoire ». Ces phrases sont tirées de son discours prononcé lors du $10^{\mathrm{e}}$ congrès du Parti Communiste en 1921 (cité dans Masenko, 2004b:19).

La reconnaissance officielle de l'égalité des deux langues, russe et ukrainien, n'a pas donné les résultats «attendus » par le pouvoir soviétique : I'ukrainien était toujours moins utilisé que le russe. En 1923, un nouveau décret sur la politique nationale en Ukraine a alors été approuvé, décret intitulé ukrainisation. Suite à cette mesure, les fonctionnaires d'État sont obligés de parler ukrainien au travail sous peine de licenciement; il est interdit d'embaucher des gens sans connaissance de la langue nationale ; I'entrée à l'université passe par un examen obligatoire d'ukrainien. 
L'ukrainisation a gagné toutes les sphères de la vie. Ainsi, la presse est devenue à $85 \%$ ukrainienne et tous les livres sont essentiellement édités en ukrainien. Subtel'nyj (1991: « Ukrainisation ») précise que l'édition de livres en ukrainien a augmenté d'environ $30 \%$ en cinq ans (1922 - 1927). Quant au nombre de périodiques en ukrainien, de 10 journaux et revues en 1922, il passe en 1933 à 373 journaux sur 426 au total. La langue du théâtre est également ukrainienne. En 1929, $80 \%$ des écoles et $30 \%$ des établissements supérieurs enseignent exclusivement en ukrainien (ibid.).

Partout en Ukraine, l'apprentissage de l'ukrainien s'intensifie : on observe des progrès dans les domaines de la linguistique, de la lexicographie et de la terminologie. La question de la normalisation de la langue littéraire est à nouveau au centre des discussions linguistiques. La création de nouveaux dictionnaires et surtout leur extension se fait par l'unification de tous les dialectes ukrainiens, y compris les dialectes occidentaux. Plus tard, dans les années 1930, cette méthode sera condamnée et traitée de «polonophilie ». Plusieurs commissions de spécialistes sont créées pour faire avancer les recherches lexicographiques. Le travail avait commencé en 1918 avec la fondation de l'Académie ukrainienne des sciences. La même année, on constitue deux commissions, dont l'une pour un dictionnaire de la langue ukrainienne vivante sous la direction de Kryms'kyj, et l'autre pour un dictionnaire historique de la langue ukrainienne sous la direction de Timčenko. En 1919, l'Académie des sciences organise une Commission de l'orthographe et de la terminologie et en 1921, elle crée I'Institut de la langue ukrainienne savante.

Entre 1924 et 1933, six dictionnaires bilingues ont ainsi vu le jour : deux ukrainien-russe et quatre russe-ukrainien. Dans son Histoire de la lexicographie ukrainienne, Horec'kyj donne des listes complètes de tous les dictionnaires publiés entre 1917 et 1933 : leur nombre approcherait 130 ouvrages, dont 83 sont des dictionnaires terminologiques (1963:172-173, 181-183).

À l'époque de I'ukrainisation, I'ukrainien est normalisé. À cette fin, une Commission de réglementation de l'orthographe ukrainienne a été créée. La réalisation de ce projet orthographique a pris beaucoup de temps. Les linguistes de la Commission ont essayé de conserver le plus possible la nature et les traditions de la langue, en s'appuyant tout de même sur la langue vivante de l'époque et sur ses différents dialectes et leur histoire. Après plusieurs discussions publiques, en 1927, la Commission organise une conférence sur l'orthographe à Kharkiv, la capitale de l'Ukraine soviétique. Cinquante représentants de toutes les régions d'Ukraine participent à cette conférence. L'objectif principal de la réunion est l'unification des deux traditions orthographiques, orientale et occidentale. Après de longs débats, le texte de L'Orthographe ukrainienne (Ukrajins'kyj Pravopys) a été écrit par Synjavs'kyj et contresigné par Skrypnyk, commissaire du peuple pour l'Éducation nationale de l'époque. Depuis, le livre est désigné comme «l'orthographe de Skrypnyk » ou «l'orthographe de Kharkiv ». Il est publié et mis en pratique en 1929.

En conclusion, il faut souligner que malgré toute l'inconséquence des démarches officielles et leur caractère inachevé, l'ukrainisation a eu une forte influence sur le changement du rôle et des fonctions de l'ukrainien dans la société : le domaine de son usage ainsi que la culture ukrainienne se 
sont considérablement élargis ; l'ukrainien est devenu, même si ce n'est que pour une courte période, la langue de l'administration étatique.

Progressivement, avec la consolidation du régime soviétique en URSS, la nécessité pour les bolcheviks d'invoquer la politique nationale dans les républiques tombe. Désormais, il faut corriger ses conséquences. C'est ainsi que tous les Ukrainiens qui ont contribué à l'ukrainisation du pays sont d'abord critiqués publiquement par ceux qui sont au pouvoir et ensuite expulsés du pays, voire disparaissent à jamais ou se suicident (Skrypnyk, Xvyl'ovyj³ et d'autres).

Un ordre de Staline met officiellement un terme à I'ukrainisation en 1933. Elle sera remplacée à nouveau par une pratique d'assimilation linguistique, mais cette fois-ci accompagnée d'une terreur inouïe.

\section{Les années 1930 : « génocide 》 linguistique}

Certains linguistes ukrainiens comme Masenko (2004b), Shevelov (1998a), les co-auteurs multiples de l'encyclopédie Ukrajins'ka mova (2004), ainsi que les auteurs du rapport de l'ECRI (Commission européenne contre le racisme et l'intolérance) sur l'Ukraine (Rapport de l'ECRI, 1999) appellent les événements qui ont suivi un génocide linguistique. Masenko, Kubajčuk et Dems'ka-Kul'čyc'ka (2005) ont donné ce nom à leur livre basé sur des archives soviétiques: La Langue ukrainienne au XXe siècle : I'histoire d'un génocide linguistique. Il s'agit d'un recueil de documents dénonçant la politique linguistique de I'URSS durant les années 1930-1970 dans les républiques soviétiques, et en Ukraine en particulier.

L'attaque des bolcheviks commence par l'Académie ukrainienne des sciences, dont presque tous les membres sont poursuivis et liquidés. Il leur est reproché une atteinte aux droits des minorités nationales, notamment des Russes. Presque toutes les recherches linguistiques des années 1920 sont décrétées préjudiciables et nationalistes, orientées vers le détachement artificiel de l'ukrainien de la langue du «frère russe ». La plupart des chercheurs ukrainiens de l'époque sont accusés de nationalisme, de thèses bourgeoises et même de fascisme ukrainien (Xvylja, 1933:passim). Tout le domaine de la linguistique, toutes les éditions scientifiques de l'Institut de la langue ukrainienne savante et de l'Institut des recherches linguistiques sont qualifiés de fascistes (Masenko, 2004b:43).

L'enseignement de l'ukrainien est rapidement réduit, tandis que l'enseignement du russe reprend ses positions antérieures. Progressivement, le premier se limite aux facultés d'ukrainien, certaines facultés de langues dans les universités, ou encore aux instituts pédagogiques. L'enseignement dans les écoles passe de plus en plus au russe, surtout dans les villes. En peu de temps, les quartiers nationaux avec des écoles enseignant dans la langue de minorité sont supprimés, et à partir de 1938 le russe devient obligatoire dans toutes ces écoles. Toutes les écoles ukrainiennes ainsi que les maisons d'édition publiant en ukrainien en dehors du pays sont fermées. Le russe redevient la langue de l'administration et commence à éliminer l'ukrainien du domaine des sciences.

\footnotetext{
${ }^{3}$ Xvyl'ovyj se suicide en mai 1933, en réaction aux attaques systématiques contre ses ouvrages ; Skrypnyk le suit en juillet de la même année, en signe de protestation contre la famine de 1932-1933 et aux arrestations des membres de l'intelligentsia ukrainienne.
} 
Cette nouvelle politique de russification rejoint bien la politique impériale de la Russie envers I'Ukraine depuis le milieu du XVII ${ }^{\text {e }}$ siècle. La différence entre les deux politiques, impériale et soviétique, repose sur les méthodes d'assimilation adoptées en raison du régime. Au XXe siècle, la russification du pays a pris une ampleur inconnue auparavant. Elle était d'autant plus dangereuse qu'elle menait l'ukrainien à sa disparition, non seulement en utilisant les méthodes classiques d'assimilation (interdiction totale ou partielle de la langue, système d'enseignement en russe, possibilité d'une carrière professionnelle seulement par le renoncement à sa propre culture, migrations dans les deux sens, etc.), mais surtout en modifiant le système même de la langue ukrainienne.

D'abord, la famine de 1932-1933 organisée en Ukraine, détruit la paysannerie ukrainienne source de la langue ukrainienne ; ensuite les bolcheviks s'attaquent à l'intelligentsia - « moteur » de son essor ; et finalement, à la langue elle-même.

C'est dans les années trente, en particulier en 1933 et 1934, que l'élan de l'ukrainien a été brisé, tant en syntaxe qu'en morphologie. Comme le proclame en 1933 le premier adjoint au commissaire du peuple pour l'Éducation nationale Andrij Xvylja, afin de « liquider l'encombrement nationaliste dans la langue ukrainienne », il faut suivre les directives suivantes :

a) arrêter immédiatement l'édition de tous les dictionnaires,

b) revoir tous les dictionnaires déjà édités et toute la terminologie,

c) effectuer une unification de la terminologie technique avec celle qui existe déjà en URSS et qui est employée en Ukraine,

d) revoir les cadres sur le front de la linguistique et bouter de ce front tous les éléments bourgeois et nationalistes,

e) revoir l'orthographe ukrainienne,

f) changer de consigne sur la présentation linguistique dans l'Encyclopédie soviétique d'Ukraine,

g) éditer un document spécial qui engloberait toutes ces questions et assurerait le développement absolu ultérieur de la culture soviétique ukrainienne [...] (Masenko \& al., 2005:130).

Toutes ces directives sont soigneusement mises en application partout dans le pays. Dès lors, certains dictionnaires sont interdits, d'autres détruits ou rendus méconnaissables par ces modifications linguistiques. Ukrajins'kyj Pravopys de 1928 est modifié et réédité en 1933. Des brigades spéciales, formées juste après, doivent revoir tous les dictionnaires terminologiques déjà interdits et changer les termes « nationalistes » contre des termes « internationaux ». Plusieurs Bulletins terminologiques sont alors publiés : tous les termes à base ukrainienne sont supprimés et remplacés par des calques du russe. Ainsi fut détruite la terminologie ukrainienne qui avait été l'objet de tant de travail dans les années 1920.

L'un des premiers ouvrages sur la situation linguistique en Ukraine au début du XXe siècle est la recherche de Shevelov, éminent linguiste slavisant du XXe siècle, $L$ 'Ukrainien dans la première moitié du XXe siècle (Shevelov, 1998a). Selon ce chercheur,

L'intrusion de l'État [...] dans les lois internes d'une langue était une invention soviétique et une grande nouveauté. Ni Polonais, ni Roumains, ni Tchèques, pas plus que l'administration tsariste de l'ancienne Russie n'y ont eu recours. Tous se sont limités à des moyens de pression extérieurs [...] (1998a:173).

Le système soviétique va, lui, beaucoup plus loin et installe un contrôle sur la structure de la langue elle-même : certains mots ainsi que des constructions syntaxiques, des formes grammaticales, des 
règles d'orthographe et d'orthoépie sont interdits. Ils sont ensuite remplacés par des variantes plus proches du russe ou carrément par des unités russes. Ainsi, le conflit entre deux langues est transféré du domaine extérieur au domaine intérieur de la langue elle-même.

Un autre ouvrage important sur le sujet est le livre déjà cité de Masenko et al. (2005). On y trouve une liste des mots réprimés par les bolcheviks. Cette liste inclut des mots et des termes ayant connu trois types de changements :

- changement total ;

- changement partiel (modification d'un préfixe ou/et suffixe) ;

- suppression complète.

Les raisons mentionnées dans le texte pour ces changements sont réduites à quelques commentaires tels que : « dialectalisme », « mot artificiel », « archaïsme », « mot polonais », « vieilli », « ne correspond plus au langage de la masse » (ibid::355). Selon le rédacteur du recueil, Masenko, ces commentaires sont souvent faux, ayant pour seul objectif le remplacement, ou même la destruction totale, des unités lexicales d'une langue (ukrainien) par celles d'une autre (russe).

Désormais, dans les dictionnaires bilingues russe-ukrainien et ukrainien-russe, une part importante du lexique ukrainien manque ou bien est déplacée en deuxième position dans l'article, après un mot proche du russe ou russifié. C'est avec ce genre de dictionnaires que les trois générations suivantes d'Ukrainiens ont appris leur langue. L'objectif de ces méthodes était clair :

[...] I'orientation forcée des ouvrages lexicographiques et autres vers un retrait progressif [...] des caractéristiques de son système autonome et sa réduction [de la langue ukrainienne] à un statut de patois local différent du russe par des particularités phonétiques et lexicales insignifiantes (Masenko, 2004b:45).

Cette politique de l'État soviétique en Ukraine reste en vigueur jusqu'à la fin des années 1980. Elle était sans cesse accompagnée de slogans sur la fraternité des deux peuples et l'influence bénéfique du russe sur la langue ukrainienne. Le rapprochement de ces deux langues fraternelles et I'harmonie du bilinguisme russe-ukrainien sont devenus les postulats des recherches linguistiques ultérieures.

\section{La politique linguistique des années 1950-1980}

Les deux périodes décrites précédemment ont déterminé la situation linguistique en Ukraine moderne et fournissent les réponses à la plupart des questions sur l'identité nationale des Ukrainiens d'aujourd'hui. Les mesures d'État des années 1950 à 1970 ne sont qu'une redite de celles du début du siècle : le «dégel » de Khrouchtchev et les dissidents des années 1960 d'abord, puis la nouvelle vague de russification à l'époque de Brejnev (1964-1982).

À l'époque du « dégel », période de relance importante des publications en ukrainien, la langue ukrainienne fut néanmoins classée, à l'instar de la plupart des langues de I'URSS, comme «nonperspective ». Outre le russe, quelques langues furent aussi classées comme «perspectives »: l'arménien, le géorgien, le lituanien, le letton et l'estonien. C'était des langues qui, tant par leur nature linguistique que par l'effet de la solidarité nationale, résistaient bien à la russification, alors que la 
parenté de l'ukrainien et du biélorusse avec le russe a condamné ces deux langues à la destruction progressive.

Ce classement mena à l'assimilation des Ukrainiens aux Russes et à la prépondérance du russe. La notion de «peuple soviétique », avec une seule et unique langue, se consolida. Khrouchtchev introduisit ensuite le concept de « deuxième langue maternelle » pour la langue russe, commune à tous les citoyens soviétiques, et de langue de communication interethnique au sein de I'URSS. L'une des mesures importantes de cette période fut la réforme de 1958, qui permit aux parents de choisir la langue d'enseignement pour leurs enfants. Les enfants pouvaient donc recevoir un enseignement en russe ou dans une langue des nationalités, telle que l'ukrainien. Dans ce dernier cas, le russe demeurait de toute façon langue obligatoire. Par la suite, pour éviter la surcharge de l'emploi du temps des élèves, le bilinguisme dans l'enseignement fut progressivement abandonné.

Les années 1960 restent toutefois une époque de velléités visant à promouvoir l'ukrainien. Plusieurs ouvrages fondamentaux sont édités : Kyryčenko (réd.), Dictionnaire ukrainien-russe (19531963) en six volumes; Holovaščuk (réd.), Dictionnaire russe-ukrainien (1969) en trois volumes; Bilodid (réd.), Dictionnaire de la langue ukrainienne (1970-1980) en onze volumes; Bilodid (réd.), Dictionnaire de la langue ukrainienne littéraire moderne (1969-1973) en cinq volumes ; Kyryljuk (réd.), Histoire de la littérature ukrainienne (1967-1971) en huit volumes ; Bažan (réd.), Histoire de l'art ukrainien (1966-1970) en six volumes; Histoire des villes et des villages de la République soviétique socialiste d'Ukraine (1962-1974) en vingt-six volumes; etc. L'édition de l'Encyclopédie ukrainienne soviétique (1959-1965), sous la rédaction de Bažan, a également débuté à cette époque, de même qu'une série de nouvelles revues sur les sciences naturelles et sociales en ukrainien.

Pourtant, un trait caractéristique des dictionnaires de cette époque fut «l'alignement » sur le russe: l'emprunt et le calque sont reconnus comme le meilleur moyen d'enrichissement du vocabulaire ukrainien. La réaction à cette nouvelle vague de russification se traduit en un mouvement dit šistedesjatnyky, «ceux des années soixante », mené par des représentants de l'intelligentsia ukrainienne orientés vers l'héritage culturel des années 1920. L'ouvrage de Dzjuba Internationalisation ou russification ? publié pour la première fois en 1965 joua un rôle important dans ce mouvement. L'auteur y désapprouve la politique nationale des époques de Staline, Khrouchtchev et Brejnev, dénonçant leur reniement des «préceptes de Lénine »(Dzjuba, 1998). Il était alors le premier à réfuter la théorie soviétique sur l'harmonie du bilinguisme russe-ukrainien. Une autre publication a également marqué l'époque, l'article d'Antonenko-Davydovyč Une lettre qui nous manque (1969), sur la disparition de la lettre $\langle\lceil>/ \mathrm{g} /$ de l'alphabet ukrainien. La suppression de cette lettre, attestée par la réforme de l'orthographe de 1933, était motivée par la complication dans l'écriture des mots et des noms propres d'origine étrangère qu'elle était censée entraîner (Antonenko-Davydovyč, 1997). Il s'agit premièrement, des mots d'origine latine et en provenance des langues européennes modernes avec le son [g], ех. : ґенерація, ґюго. En revanche, dans les mots d'origine grecque ainsi que dans les emprunts étrangers modernes avec le son [ $\mathrm{\hbar}]$, on écrit la lettre $\langle\Gamma>$, еx. : географія, Геродот, гусар. 
Le deuxième groupe de mots avec $\langle\lceil\rangle$ initial ou médian est composé d'unités purement ukrainiennes. Le dictionnaire de Hrinčenko (1907-1909) en cite 270.

Les šistedesjatnyky ont principalement réagi dans deux directions :

- Contre la russification extérieure et la réduction des fonctions sociales de la langue ukrainienne.

- Contre l'intrusion, planifiée par le régime soviétique, dans le développement intérieur de la langue, notamment dans ses systèmes grammatical et lexical.

Le régime ne leur a pas pardonné : au début des années 1970 une vague massive d'arrestations politiques frappe les dissidents. Beaucoup d'entre eux se retrouvent en prison, en exil, ou expulsés.

De nouveau, l'ukrainien est chassé du système éducatif, des sciences, des médias. En 1983, le gouvernement prend un décret sur le renforcement de l'apprentissage de la langue russe à l'école. Les classes dans les établissements ukrainiens sont désormais divisées en deux et les enseignants de russe voient leur salaire augmenté de $15 \%$ (Masenko, 2004b:71). Le problème du bilinguisme en Ukraine est déclaré résolu : même si le russe est majoritairement parlé par les Ukrainiens, cela n'empêche pas les autorités de l'appeler « seconde langue maternelle » des Ukrainiens.

Quant à la lexicographie et la terminologie en particulier, depuis les Bulletins terminologiques des années 1934-1935, le principe des emprunts et des calques du russe s'est fortement installé dans le domaine de la création des systèmes terminologiques ukrainiens, principe difficile à bouleverser, même aujourd'hui. Le lexique de l'Ouest, disparu des dictionnaires soviétiques, et dans lequel les traducteurs et les linguistes des années 1960-1970 puisaient leur inspiration, fut proclamé archaïque ou dialectal; et tout emploi de ce vocabulaire défendu subissait une censure sévère de la normalisation soviétique. Ainsi, les maisons d'édition et les rédactions des journaux recevaient des listes de mots censurés en plus des listes des auteurs interdits existant depuis les années 1930.

Shevelov attribue les caractéristiques suivantes à l'ukrainien de cette époque :

- large ouverture vers des inclusions et des structures russes ;

- attribution d'une échelle russe des valeurs sociales dans la langue ;

- fermeture aux emprunts autres que ceux qui viennent de, ou passent par le russe ;

- sous-développement de la terminologie technique et scientifique ;

- manque d'argot urbain ;

- Phénomène de suržyk ${ }^{4}[\ldots]$.

- [...] [Or, ] la langue ukrainienne en Ukraine soviétique demeure dans un état intermédiaire entre la langue et le patois (Shevelov, 1998b).

Dans ces conditions, l'usage de l'ukrainien était considéré par beaucoup comme un trait caractéristique d'individus incultes. Son utilisation se limitait à la sphère familiale, à certaines œuvres originales de la littérature ukrainienne, à certaines publications en sciences humaines (surtout la philologie ukrainienne), aux écoles maternelles et primaires en Ukraine occidentale, à quelques

\footnotetext{
${ }^{4}$ Mélange artificiel et sans respect des normes littéraires des élements de deux langues, ukrainien et russe en I'occurence, nocif pour les deux langues. Pour une étude détaillée de ce phénomène linguistique, voir Shevchenko (2011:134-146).
} 
journaux et revues, et à la radio locale. Le cinéma, considéré par les communistes comme l'art le plus important par son influence sur les masses, n’a jamais été ukrainophone.

\section{Indépendance et pratique des langues en Ukraine d'aujourd'hui}

Les conséquences de cette politique dévastatrice sont encore visibles aujourd'hui.

Lors du recensement de 1989, $73 \%$ de personnes se déclarent de nationalité ukrainienne, $22 \%$ de nationalité russe et $5 \%$ se disent appartenir à d'autres minorités. Dans ce même recensement, la question sur la langue maternelle a également été posée.

Il faut préciser qu'en ukrainien, la question de la langue maternelle demeure parfois problématique vu l'ambiguïté de sa notion même en ukrainien - ridna mova. Pour certains, il s'agit non pas de la langue la mieux maitrisée ou la plus souvent utilisée, ou encore, la langue de la mère (comme on pourrait le définir en français) mais d'une langue que la personne interrogée considère comme maternelle par son appartenance à une communauté linguistique donnée. C'est-à-dire, une langue de la nation.

L'ambiguïté de l'expression en ukrainien amène à l'imprécision des données statistiques sur la maîtrise de cette langue dans la société moderne. Ainsi, lors du recensement de 1989, 64 \% des personnes interrogées déclarent l'ukrainien comme leur langue maternelle, tandis que seulement $44 \%$ indiquent préférer s'exprimer en ukrainien. Ces $20 \%$ de décalage témoignent d'un phénomène qu'on appelle en sociolinguistique loyauté linguistique, c'est-à-dire une «fidélité » linguistique du locuteur, son sentiment d'appartenance à une communauté linguistique donnée. Et même ce chiffre de $44 \%$ me paraît excessif car il témoigne plus du sentiment d'appartence à une nation que d'une maitrise de la langue ukrainienne à cette époque.

La même année, en 1989, I'Ukraine adopte une loi sur les langues ${ }^{5}$ qui déclare l'ukrainien seule langue officielle du pays. Une loi qui a tout de même de nombreuses contradictions. Notamment I'article 3 qui approuve l'utilisation dans les sphères sociales importantes de toute langue employée par une minorité ethnique, comme par exemple les Russes, et majoritaire à l'échelle locale (oblast, ville, village, etc.). Ainsi, le russe devient dans presque tous les cas une alternative, en particulier dans les régions majoritairement russophones.

Les domaines dans lesquels l'État peut prescrire l'emploi de la langue officielle et soutenir ainsi la formation d'une nation sont avant tout les médias, l'enseignement, l'administration et l'armée. En 1989, par cette loi sur les langues, l'ukrainien est déclaré obligatoire seulement dans les domaines suivants :

- Les annonces officielles et les communiqués, les affiches, les publicités (la traduction dans une autre langue peut cependant être rajoutée) (article 35).

- Les désignations des marchandises, les étiquettes et les modes d'emploi pour les produits fabriqués en Ukraine (article 36).

\footnotetext{
${ }^{5}$ Loi sur les langues en RSS d'Ukraine du 28.10.1989 № 8312-XI.
} 
- Les dénominations officielles des organes étatiques et publics et des partis, des entreprises, organisations et établissements (avec traduction possible dans une autre langue) (article 37).

- Les toponymes (article 38).

Après l'indépendance, la question linguistique devient un enjeu important pour l'Etat ukrainien, qui tente de s'affirmer par ce biais. Dans les années 1990, plusieurs projets de loi consacrés aux langues ont été proposés mais la plupart ont été jugés non conformes à la Constitution par la Cour constitutionnelle.

La Constitution ukrainienne, adoptée le 28 juin 1996, par la Verxovna Rada, le Parlement ukrainien, affirme la position de l'ukrainien comme seule langue officielle du pays. Elle obtient un statut de deržavna mova, équivalent de langue d'État en français. Selon la décision de la Cour constitutionnelle de I'Ukraine (article 3 de cette décision), les deux termes langue officielle et langue d'État désignent la même notion :

La langue d'État (langue officielle) est une langue à laquelle l'État attribue un statut juridique de moyen obligatoire de communication dans les lieux publics de la vie sociale. La Constitution d'Ukraine attribue ce statut à la langue ukrainienne [...] (VRUZ : Constitution).

En réalité, ils sont souvent utilisés pour différencier la langue d'État des langues des minorités.

Ainsi, par exemple, dans la Constitution de la république autonome de Crimée adoptée en 1992, deux langues ont obtenu le statut de langues d'Etat - l'ukrainien et le tatar de Crimée. Le russe a été désigné « langue officielle », statut que la loi ne prévoyait pas. Dans la dernière Constitution de Crimée avant l'annexion par la Russie, celle de 1998, approuvée par le président de l'Ukraine, l'ukrainien était unique langue d'Etat, le tatar et le russe ont perdu leur statut officiel mais le russe a été qualifié de «langue de la population majoritaire employée dans toutes les sphères de la vie sociale » (VRUZ : Constitution de la République autonome de la Crimée adoptée le 21.10.1998, article 10).

A Odessa, il a été déclaré «langue officielle » en 1993. Dans les régions de Donetsk et de Louhansk, le russe est depuis 1994 « la deuxième langue d'Etat », statut encore une fois, que la loi ne prévoyait pas. Depuis 1994, les parlements de Donetsk, Kharjiv, Mykolaïv, Horlivka et Kharcyz'k ont introduit le russe comme « langue officielle » ou comme « deuxième langue d'Etat ».

Dans les années 2000, le gouvernement accorde une grande importance à la langue ukrainienne dans la définition de l'identité nationale : plusieurs lois renforçant la position de l'ukrainien dans la société sont alors approuvées, surtout pendant le mandat du président Iouschenko (20052010).

En 2006, l'article 10 de la Loi sur la radiodiffusion et la télévision ${ }^{6}$ fixe la part de la diffusion nationale en ukrainien à $75 \%$ du volume quotidien. Depuis, sur toute chaîne, publique ou privée, un film ou une émission dans une autre langue que l'ukrainien doit être obligatoirement doublé en langue nationale. Dans la pratique, le doublage à la télévision est quelquefois remplacé par le sous-titrage ou la traduction simultanée. Il arrive que la télévision ukrainienne propose des émissions en deux langues avec des propos en russe et en ukrainien. On rencontre également du bilinguisme spontané, quand

\footnotetext{
${ }^{6}$ Loi du 12.01.2006 № 3317-IV.
} 
les questions du journaliste sont en ukrainien et les réponses de son interlocuteur sont en russe. Une telle « cohabitation » linguistique entraîne la suržykisation, un mélange nocif pour les deux langues.

Si l'ukrainien domine sur les chaînes de télévision et de radio publiques, ce n'est toujours pas le cas dans les médias privés: presque deux tiers du temps total de diffusion est en russe. Concernant la presse écrite, seulement $23 \%$ des revues et $27 \%$ des journaux sont publiés en ukrainien (Annuaire des statistiques de l'année 2000).

Le domaine du livre en ukrainien se trouve dans un état déplorable. Toujours en 2000, les librairies locales affichent $90 \%$ de la production russe.

Quant au cinéma, depuis le $1^{\text {er }}$ juillet 2007 , au moins $70 \%$ de la production étrangère doit être doublée ou sous-titrée en ukrainien, que ce soit les films à projeter dans les salles, à la télévision ou chez soi.

Cette politique linguistique a porté ses fruits. Si en 2002, selon les données du Centre Razumkov (UCEPS = Ukrainian Centre for Economic and Political Studies), seulement 37,5\% des Ukrainiens se disent communiquer en ukrainien à la maison (Figure 1). En 2011, toujours selon UCEPS, ils sont 53,3\%. La même tendance se voit dans le milieu de travail : ils étaient $34 \%$ à communiquer en ukrainien en 2002 (Figure 2), et ils le sont à 49,2 \% en 2011. Les autres parlent le russe ou bien mélangent les deux (20\% en 2002). L'un des traits caractéristiques de la situation linguistique en Ukraine d'aujourd'hui est donc un bilinguisme ukrainien-russe.

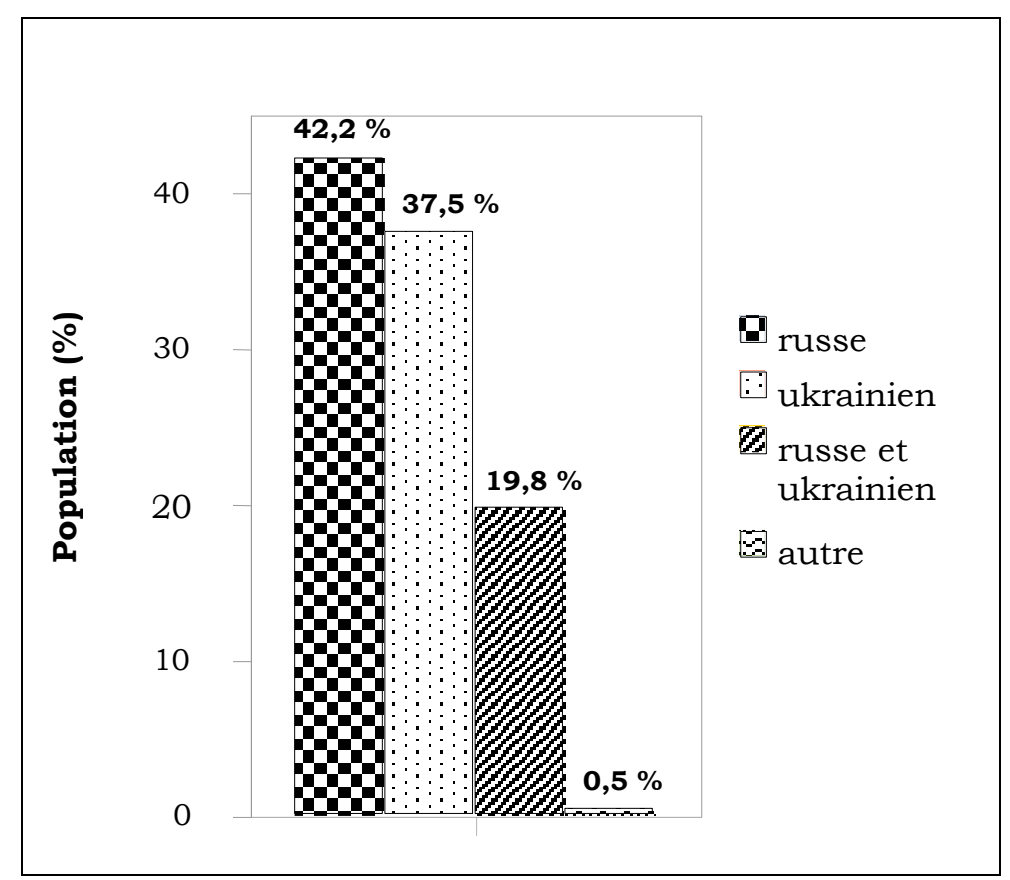

Figure 1 : Langue de communication à la maison Source : Sondage réalisé en décembre 2002 par I'UCEPS 


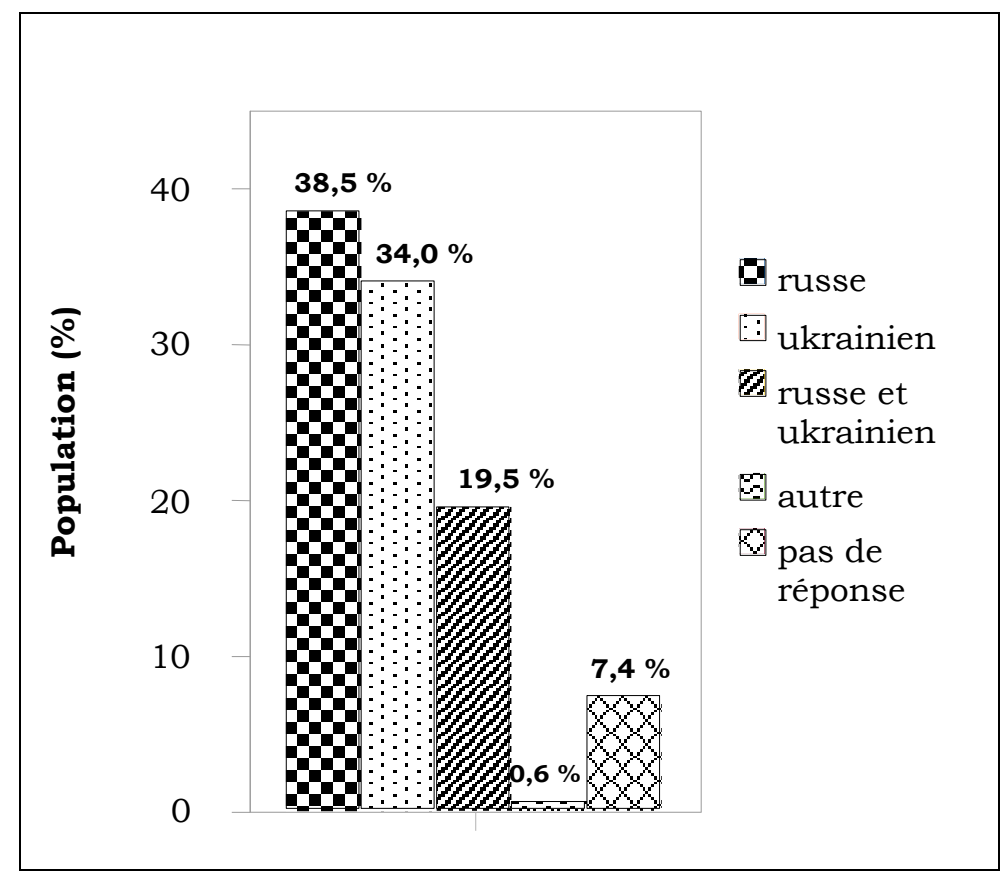

Figure 2 : Langue de communication au travail

Source : Sondage réalisé en décembre 2002 par I'UCEPS

Une question fréquemment posée quand on parle du bilinguisme russe et ukrainien porte sur le degré de similitude entre ces deux langues. Du point de vue lexical, l'ukrainien et le russe coïncident à 70,5 \%, selon l'étude de Koptilov (1995:72) ; tandis que la coïncidence entre le français et l'espagnol, par exemple, est de $75 \%$, celle entre le français et l'italien de $89 \%$, selon les données rassemblées par Lewis (2009) 7 .

Un phénomène linguistique propre à l'Ukraine est le suržyk. C'est un mélange de deux langues qui est très répandu dans le pays avec des pourcentages différents suivants les régions et surtout les milieux sociaux. Il n'y a pas de statistiques officielles sur le sujet, mais on estime qu'un cinquième de la population parlerait ce mélange que certains linguistes par conviction ou d'autres par provocation proposent de reconnaître comme langue à part entière et même de l'installer comme une des langues d'Etat (Irvanec cité dans Ul'janov, 2004).

Il est important à souligner qu'à l'inverse des situations habituelles connues en Europe, c'est l'ethnie majoritaire - les Ukrainiens - qui a donc besoin du soutien des autorités pour imposer la langue officielle. C'est la langue d'État qui devrait être protégée comme «langue minoritaire ». Le modèle « classique », d'un État avec une nation dominante et une ou plusieurs minorités ayant besoin d'une protection particulière, ne correspond pas à la réalité ukrainienne.

Il est possible qu'avec le temps l'Ukraine puisse héberger les deux ethnies principales du pays, les Ukrainiens et les Russes, à l'égal l'une de l'autre ; on pourrait alors penser à un modèle bilingue pour l'État ukrainien. Mais d'abord, il importe de permettre à la nation ukrainienne de se définir et se renforcer, jusqu'à devenir suffisamment solide et indépendante pour pouvoir trouver sa puissance

\footnotetext{
${ }^{7}$ Chapitre: «French. A language of France ».
} 
dans la diversité. L'Ukraine devra alors admettre l'idée d'une nation politique, et non pas d'une nation ethnique. La diversité culturelle doit être respectée.

La problématique du statut des langues minoritaires, et en particulier du russe, resurgit à chaque échéance électorale. La question linguistique devient un enjeu de pouvoir, qui est mobilisé aussi bien par les partisans du russe que de l'ukrainien, et indépendamment de leur langue maternelle ou de leurs préférences politiques.

Pourtant, pour la majorité de la population, la question linguistique à côté des problèmes économiques, politiques et sociaux n'a jamais eu une grande importance. Seuls les responsables politiques et les intellectuels en débattent régulièrement.

Ainsi, le Président Ianoukovytch intègre dans son programme électoral la question linguistique en promettant à ses électeurs de donner le statut de deuxième langue d'Etat pour le russe. Il ne l'a pas fait quand il a été élu en 2010.

Mais le 3 juillet 2012, le Parlement ukrainien adopte une loi (approuvée le 10 août 2012) sur les langues permettant I'utilisation dans toutes les sphères sociales d'une « langue régionale » ou «langue minoritaire » dans les régions où les minorités ethniques dépassent les $10 \%$ de la population. Suite à cette loi, 13 sur 27 régions ukrainiennes deviennent officiellement bilingues, toutes avec le russe comme langue régionale malgré le fait que le texte de la loi concerne 18 langues minoritaires.

En 2014, suite aux événements révolutionnaires de la fin de 2013 et du début de 2014, le président par interim Oleksandr Tourtchynov à peine arrivé au pouvoir le 23 février 2014 fait voter le jour même par le Parlement ukrainien la suppression de cette loi sur les langues. Une erreur qui a tout de suite provoqué une vague de mécontentements dans la population des régions du Sud et de l'Est de I'Ukraine. Oleksandr Tourtchinov revient vite alors au texte de 2012.

Cependant, cette décision précipitée donne au président russe le prétexte d'une défense de la population russe en Ukraine : en Crimée d'abord et dans les régions de l'Est ensuite.

En conclusion, les événements de la fin de 2013-début 2014 ont signalé une nouvelle étape dans l'évolution de l'Etat ukrainien. L'idée de la nation ethnique nourrissant la révolution orange, est remplacée par l'idée d'une nation politique. Le pays se retrouve à nouveau face au défi de la multiculturalité dans un processus de formation d'identité nationale qui est loin d'être achevé. Dans la conscience de la population, la langue n'est plus le trait principal de l'identité ukrainienne. La formation d'une identité ukrainienne passe d'une définition en termes ethniques à une définition en termes politiques.

\section{Bibliographie}

1. Antonenko-Davydovyč, В. D. - Антоненко-Давидович, Б. Д. (1997). Як ми говоримо. (Comment parlons-nous). Київ : Українська книга.

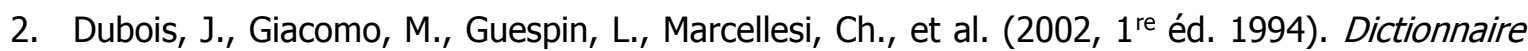
de linguistique. Paris : Larousse. 
3. Dzjuba, I. М. - Дзюба, I. М. (1998). Інтернаціоналізм чи русифікація? (Internationalisation ou russification ?). Київ : КМ Академія. <http://litopys.org.ua/idzuba/dz.htm> [page consultée le 11 novembre 2014].

4. Horec'kyj, Р. J. - Горецький, П. Й. (1963). Історія української лексикографії. (Histoire de la lexicographie ukrainienne). Київ : Видавництво АН Української РСР.

5. Hrinčenko, В. - Грінченко, Б. (1907-1909). Словарь української мови. (Dictionnaire d'ukrainien). <http://r2u.org.ua/html/hrinchenko_pro.html> [page consultée le 11 novembre 2014].

6. Koptilov, V. (1995). Parlons ukrainien : Langue et culture. Paris : L'Harmattan.

7. Lacorne, D., Judt, T. (dirs). (2002). La Politique de Babel: Du monolinguisme d'État au plurilinguisme des peuples. Paris : Karthala.

8. Leclerc, J. (2007). Les langues slaves. In L'Aménagement linguistique dans le monde. Québec: TLFQ, université Laval. <http://www.tlfq.ulaval.ca/axl/monde/langues slaves.htm> [page consultée le 11 novembre 2014].

9. Lewis, M. Paul (ed.). (2009, $16^{\text {th }}$ ed.). Ethnologue : Languages of the World. Dallas, Tex. : SIL International. <http://www.ethnologue.com/country/UA> [page consultée le 11 novembre 2014].

10. Malherbe, M. (1995). Les Langages de l'humanité: Une encyclopédie des 3000 langues parlées dans le monde. Paris : R. Laffont.

11. Masenko, L. - Масенко, Л. (2004а). Мова і політика. (Langue et Politique). Київ : Соняшник.

12. Masenko, L. - Масенко, Л. (2004b). Мова і суспульство : Постколоніальний вимір. (Langue et Société : Dimension postcoloniale). Київ : КМ Академія.

13. Masenko, L. (dir.), Kubajčuk, V., Dems'ka-Kulčyc'ka, O. - Масенко, Л., Кубайчук, В., Демська-Кульчицька, О. (2005). Українська мова у XX сторіччі : історія лінгвоциту. Документи і матеріали. (La Langue ukrainienne au XXe siècle: Histoire d'un génocide linguistique. Documents et matériaux). Київ : КМ Академія.

14. Rapport de l'ECRI (1999) = Commission européenne contre le racisme et l'intolérance. Approche pays-par-pays de I'ECRI: Rapport sur I'Ukraine. Strasbourg. <http://www.coe.int/t/dghl/monitoring/ecri/default_fr.asp> [page consultée le 11 novembre 2014].

15. Shevchenko, N. (2011). Lexicographie bilingue français-ukrainien des unités de bas niveau de formalité. Thèse de doctorat. Université Lumière Lyon 2.

16. Shevelov, George Y. - Шевельов, Ю. (1998а). Українська мова в першій половині ХХ ст. (1900-1941). Стан і статус. (L'Ukrainien dans la première moitié du XXe siècle. (1900-1941). Etat et statut). Чернівці : Рута.

17. Shevelov, George Y. (1993). Ukrainian. In Comrie, B., Corbett, G. G. (eds). The Slavonic languages. 947-998. London, New York : Routledge.

18. Shevelov, George Y. (Šerex) - Шевельов, Ю. В. (Шерех) (1998b). Поза книжками і з книжок. (Au-delà et à partir des livres). Київ : Час. <http://r2u.org.ua/node/111> [page consultée le 11 novembre 2014].

19. Subtel'nyj, О. - Субтельний, О. (1991). Історія України. (Histoire de I'Ukraine). <http://studentbooks.com.ua/content/view/119/49/> [page consultée le 11 novembre 2014].

20. UCEPS = Ukrainian Centre for Economic and Political Studies named after Olexander Razumkov. Український центр економічних і політичних досліджень імені Олександра Разумкова. <http://razumkov.org.ua/ukr/socpolls.php?cat_id=174> [page consultée le 11 novembre 2014].

21. Ukrajins'ka mova (2004) = Українська мова. Енциклопедія. (Langue ukrainienne: Encyclopédie). Київ : Українська енциклопедія ім. М.П.Бажана.

22. Ul'janov, А. - Ульянов, А. (2004). Суржик - язык нации или свинопасов? (Le suržik langue de la nation ou des porchers ?) <http://www.proza.com.ua/events/surzhik_jazyk_natsii_ili_svinop_4a62.shtml> [page consultée le 18 août 2008]. 
23. VRUZ = Верховна Рада України. Законодавство. (Parlement ukrainien, législation). $<$ http://zakon.rada.gov.ua/> [page consultée le 11 novembre 2014].

24. Xvylja, А. - Хвиля, А. (1933). Викорінити, знищити націоналістичне коріння на мовному фронті. (Redresser, détruire les racines nationales dans le domaine linguistique). In Masenko, L. (dir.), Kubajčuk, V., Dems'ka-Kulčyc'ka, O. - Масенко, Л., Кубайчук, В., ДемськаКульчицька, О. (2005). Українська мова у ХХ сторіччі : історія лінгвоциту. Документи і матеріали. (La Langue ukrainienne au XXe siècle: L'histoire d'un génocide linguistique. Documents et matériaux). Київ : КМ Академія. 